\title{
Economic feasibility assessment of one and two stages dry fractionation of palm kernel
} oil.

\begin{abstract}
Palm kernel oil can be fractionated into stearin and olein fractions. Traditionally, industrial players only fractionate the softer stearin fraction and harden it through complete hydrogenation. One stage dry fractionation yielded $37 \%$ of softer or $24 \%$ of harder stearin fraction. Two stages dry fractionation yielded $24 \%$ harder and $11 \%$ softer stearin fractions. Even though two stages dry fractionation salvaged up to $14 \%$ of the softer stearin fraction from the normally discounted palm kernel olein fraction, the double fractionation process was not always commercially feasible. Deciphering actual price statistics revealed that on a per metric tonne basis, the refining premium ranged from MYR90 to 250. Palm kernel olein discount was as high as $9 \%$ but could command premium over crude PKO by up to $2.5 \%$. Due to the intrinsic relationship, the corresponding palm kernel stearin breakeven value could range from discount of MYR90 to premium of MYR1260 over crude PKO. The actual 60 months average market premium for palm kernel stearin stood at MYR870, promising an average MYR430 returns to refiners and processors. By incorporating two stages dry fractionation when the PKL discount was greater than $2.43 \%$, refiners and processors could boost their net margins. When the PKL discount was 9\%, the net margin could be boosted by up to $16 \%$.
\end{abstract}

Keyword: Dry fractionation, Mathematical model,Feasibility assessmenta 